\title{
The De Jong Gierveld short scales for emotional and social loneliness: tested on data from 7 countries in the UN generations and gender surveys
}

\author{
Jenny De Jong Gierveld • Theo Van Tilburg
}

Received: 20 May 2009/Accepted: 22 March 2010/Published online: 9 April 2010

(C) The Author(s) 2010. This article is published with open access at Springerlink.com

\begin{abstract}
Loneliness concerns the subjective evaluation of the situation individuals are involved in, characterized either by a number of relationships with friends and colleagues which is smaller than is considered desirable (social loneliness), as well as situations where the intimacy in confidant relationships one wishes for has not been realized (emotional loneliness). To identify people who are lonely direct questions are not sufficient; loneliness scales are preferred. In this article, the quality of the three-item scale for emotional loneliness and the three-item scale for social loneliness has been investigated for use in the following countries participating in the United Nations "Generations and Gender Surveys": France, Germany, the Netherlands, Russia, Bulgaria, Georgia, and Japan. Sample sizes for the 7 countries varied between 8,158 and 12,828. Translations of the De Jong Gierveld loneliness scale have been tested using reliability and validity tests including a confirmatory factor analysis to test the two-dimensional structure of loneliness. Test outcomes indicated for each of the countries under investigation reliable and valid scales for emotional and social loneliness, respectively.
\end{abstract}

Keywords Loneliness scale - Emotional loneliness · Social loneliness $\cdot$ Partner status $\cdot$ Health

\footnotetext{
J. De Jong Gierveld ( $₫$ )

Netherlands Interdisciplinary Demographic Institute (NIDI),

The Hague, Netherlands

e-mail: gierveld@nidi.nl

T. Van Tilburg

Faculty of Social Sciences, VU University, Amsterdam,

The Netherlands
}

Social embeddedness in the realms of the family and the broader community and alleviation of loneliness are central issues in the construction of social well-being and quality of life. This is true for younger as well as older people, and justifies the ongoing interest of social scientists in investigating the social bonds of individuals in different phases of the life course, as well as identifying the mechanisms responsible for creating either social embeddedness or loneliness. Loneliness is a universal phenomenon, but the antecedents vary, to a large extent based on personal and contextual determinants (De Jong Gierveld et al. 2006). Loneliness is a subjective and negative experience, and is the outcome of the subjective, cognitive evaluation of there being a mismatch between the quantity and quality of existing relationships on the one hand, and relationship standards on the other (Perlman and Peplau 1981). Loneliness is but one of the possible outcomes of the evaluation of a situation characterized by a small number of relationships. Where a person appears along the subjective loneliness continuum depends on his or her relationship standards. Dykstra and De Jong Gierveld (1994) showed, for example, that the degree to which widowed adults experienced loneliness depended, among other factors, on their "partner standard"; the more importance placed on having a partner, the more lonely the widowed were.

Many factors, among which socioeconomic ones (education, income), physical and mental health, and social roles (being a spouse, parent) are more or less directly associated with the size, composition and perceived quality of one's social network, and through these variables associated with loneliness (Hawkley et al. 2008). Some of the determinants of loneliness, such as the deaths of the partner and of peers, deteriorating health and financial pressures, are directly related to events and transitions in later phases of life. Therefore, research into loneliness of older adults is 
especially important. Policy makers are interested to know more about both the social embeddedness and loneliness of adults. Additionally, they may want to compare the situation of adults in their own country with adults in neighboring countries in order to investigate the similarities and differences in social characteristics and the outcomes of relevant social policies in these countries.

To answer questions on differences in embeddedness and loneliness between countries reliable and valid measuring instruments are needed. This article addresses the loneliness measurement as used in the United Nations Generations and Gender Surveys (GGS). In 2000, the Population Activities Unit (PAU) of the UN Economic Commission for Europe (UNECE) launched the Generations and Gender Programme. The Programme is a system of national Generations and Gender Surveys and contextual databases. The main substantive goal of the Programme is to improve understanding of the factors that influence demographic development. By the end of 2008, ten countries had completed the fieldwork for the first wave of the panel surveys. For each country face-to-face interviews with a random sample of the population aged 18 to 79 years had been completed. Main themes in the survey questionnaire were: the descriptive and explanatory factors of social and demographic developments, with particular attention given to relationships between adult children and parents (generations) and relationships between partners (gender). For more information, see Vikat et al. (2007). Included in the questionnaire was a module about health and well-being, encompassing among other things a three-item emotional loneliness scale, and a three-item social loneliness scale. Data from the loneliness scales were available for respondents in seven countries: France, Germany and the Netherlands from Western Europe, Bulgaria, the Russian Federation and Georgia from Eastern Europe, and Japan. Demographically the countries differed significantly, especially where the ageing of the populations was concerned; moreover, socioeconomic indicators revealed sharp differences in economic well-being between countries (see Table 1).

Before starting comparative research, we need to know the extent to which measuring instruments for emotional and social loneliness are useful tools in this context. We formulated our research questions as follows:

Are the 3-item emotional and the 3-item social loneliness scales as translated for use in different countries, reliable and valid measuring instruments? Are the associations of loneliness with several factors indicative of general mechanisms that operate in various countries more or less in a similar way?

In this article, the outcomes of tests investigating both the reliability and validity of the emotional and social loneliness scales are reported, in order to provide fellow researchers with solid information about the usefulness of the scales for culturally and economically divergent countries. It is often thought that loneliness is a problem present specifically among older adults; however, this assumption finds only limited support (Dykstra 2009). We, therefore, studied both older and younger adults.

\section{The concept of loneliness}

Loneliness has to be differentiated from social isolation which denotes the objective characteristics of a situation and refers to the absence of relationships with other people (Cornwell and Waite 2009). Investigating social isolation requires the identification of the objective characteristics of the functioning of communities, such as help in neighborhoods, and the size, composition and functioning of someone's network of personal relationships (Van Tilburg 1998). The continuum of objective social isolation puts social isolation at one extreme and social participation at the other. Loneliness, however, reflects an individual's subjective, cognitive evaluation of his or her social participation, or social isolation, against the standards held for optimal embeddedness in a social network. Loneliness is considered to be an expression of negative feelings that can manifest itself in individuals of all ages. The opposite of loneliness is feeling embedded.

Two components of loneliness can be distinguished. Weiss (1973) differentiated emotional loneliness, related to the absence of an intimate relationship (partner, best friend), and social loneliness, related to the absence of a broader, engaging social network (siblings, cousins, friends, and neighbors). Emotional loneliness arises, for example, when a partner relationship dissolves through divorce or being widowed, and is characterized by intense feelings of emptiness, abandonment, and forlornness. People who have moved to a place where they are newcomers frequently report social loneliness.

\section{Measuring loneliness}

The De Jong Gierveld 11-item loneliness scale (De Jong Gierveld and Kamphuis 1985; De Jong Gierveld and Van Tilburg 1999) can be applied as a unidimensional loneliness scale, but the items were developed with Weiss's (1973) distinction between social and emotional loneliness in mind. For that reason, researchers can-depending on the research question-choose to use either the complete loneliness scale, or the emotional (six items) and social (five items) subscales.

For use in large surveys a shorter 6-item version of the De Jong Gierveld scale was constructed in such a way that the threefold application of the original scale (an overall loneliness scale as well as emotional and social subscales) was still guaranteed. In selecting the three items for emotional loneliness out of the original set of six and the three 
Table 1 Demographic and financial indicators and the GGS sample size of the countries under investigation

\begin{tabular}{|c|c|c|c|c|c|c|c|}
\hline & France & Germany & Netherlands & Russia & Bulgaria & Georgia & Japan \\
\hline Population size (in thousands) & 60,940 & 82,728 & 16,429 & 141,900 & 7,615 & 4,395 & 128,325 \\
\hline \multicolumn{8}{|l|}{ Percentage population aged $60+$} \\
\hline Female & 24 & 28 & 22 & 21 & 26 & 21 & 31 \\
\hline Male & 19 & 22 & 18 & 13 & 20 & 15 & 25 \\
\hline \multicolumn{8}{|l|}{ Percentage population aged $80+$} \\
\hline Female & 7 & 7 & 5 & 4 & 4 & 3 & 7 \\
\hline Male & 3 & 3 & 2 & 1 & 2 & 2 & 4 \\
\hline \multicolumn{8}{|l|}{ Life expectancy at birth } \\
\hline Female & 83.5 & 82.1 & 81.6 & 71.8 & 76.3 & 74.8 & 86.4 \\
\hline Male & 76.5 & 76.3 & 76.3 & 58.7 & 69.8 & 67.1 & 79.1 \\
\hline \multicolumn{8}{|l|}{ Life expectancy at age 60} \\
\hline Female & 26.0 & 24.5 & 24.2 & 19.2 & 20.1 & 20.4 & 28.1 \\
\hline Male & 20.9 & 19.9 & 19.6 & 13.9 & 16.3 & 16.7 & 22.3 \\
\hline GDP per capita (USD) & 35,375 & 34,955 & 40,535 & 6,877 & 3,956 & 1,746 & 34,661 \\
\hline Sample Size GGS & 10,069 & 9,604 & 8,158 & 11,261 & 12,828 & 10,000 & 9,074 \\
\hline
\end{tabular}

Notes: Source for the population size and life expectancy: World Population Ageing (2007). New York, United Nations, Population Division. GDP is the Gross Domestic Product. Source: United Nations, Statistical Division (June 2008)

items for social loneliness out of the original five, the items with the highest factor loadings were selected. Subsequently, the selection procedure was oriented toward addressing a broad range of item difficulties. Following this, one of the selected items was replaced by the item scoring fourth on the rotated factor; the shorter version of the scale has been developed and tested for use in the Netherlands (for more information see De Jong Gierveld and Van Tilburg 2006). As stated above, the aim of the current study was to test the reliability and validity of the emotional and social subscales, for use in different countries.

\section{Prediction of loneliness}

Many determinants work together in explaining why some people with a small number of social contacts or a nonsatisfying quality of contacts consider themselves to be lonely, whereas others feel good and sufficiently embedded. In this investigation of the quality of the loneliness scales, a selection of loneliness predictors will be used to test the congruent validity in countries from different regions of the world. As far as emotional loneliness is concerned, the presence of an intimate partner is of crucial importance for young and old adults. Especially in later phases of the life course, after retirement and children leaving home, older adults become more reliant on their immediate social environment, in particular, on the bond with the co-resident partner (Carstensen 1992; Dykstra and Fokkema 2007; Stevens and Westerhof 2006; Waite and Gallagher 2000). We investigated, therefore, the relationship between emotional loneliness and the presence of a co-resident partner.
The experience of social loneliness is primarily related to the evaluation of deficiencies in the broader network of social relationships, including the size and composition of the social network and the presence of children in the network. Especially in later life, having no children is a major risk factor for social loneliness (Buber and Engelhardt 2008; Pinquart 2003). In testing congruent validity, we investigated social loneliness for adults with no, or a small number of children, and adults with more children.

Both the emotional and social loneliness have proved to be associated with physical health conditions at both the younger and later ages. Research has shown that poor vision and hearing, having lung disease or arthritis, and poor health conditions in self and spouse (Korporaal et al. 2008; Penninx et al. 1999; Savikko et al. 2005) contributed to increased levels of loneliness. In testing the congruent validity of the emotional and social loneliness scales we compare, in each of the 7 countries, adults confronted with poor physical health with adults in more optimal health conditions.

Low levels of income, and especially difficulties in making ends meet in a household, have been associated with chronic stressful situations (Savikko et al. 2005), the experience of being disadvantaged, less opportunities to engage in joyful activities with confidants and other members of the social network, and with emotional and social loneliness (Hawkley et al. 2008). Therefore, adults confronted with difficulties in making ends meet are compared to adults without financial pressures to investigate differences in the intensity of emotional and social loneliness in each of the countries under investigation. 
In analyzing the congruent validity of the two loneliness scales, we take on board two background variables: age and gender. Age and loneliness are correlated in Western countries, but multivariate analyses showed additionally, that an association between age and social or emotional loneliness, especially at ages of 60 and above, is - to a large extentmediated by health, financial pressures, and absence of a partner in the household (De Jong Gierveld et al. 2009). After taking these variables into account, the association between age and emotional and social loneliness was shown to decrease. Age differences in emotional and social loneliness are investigated for each of the countries. Gender differences in loneliness were taken into account but only for social loneliness. Research has shown the specific role of women as kin keepers and as the ones who take the initiatives to guarantee ongoing contacts with kin and non-kin members of the broader social network (Korporaal et al. 2008). In the context of testing congruent validity, the association between gender and social loneliness will be investigated.

\section{Methods}

\section{Respondents}

For the investigation of the reliability and validity of the loneliness scales, data have been analyzed from seven countries involved in the GGS. Although varying types of research institutes have been involved in the fieldwork phase, for e.g., country statistical institutes, demographic institutes, and others, guidelines have been developed centrally, oriented toward country representative samples. The guidelines encompassed the use of probability sampling of the resident, non-institutionalized population aged 18 to 79 years. It was recommended that countries minimized exclusions from the target population to less than $5 \%$ of the target population. Units had to be randomly selected and all units needed to have a non-zero inclusion probability in the sample. In using either a survey list or an area frame, the following auxiliary variables were needed for each person: age, sex, place of residence, and contact information. For the age group 18-44 years, equal numbers of men and women were requested; the total sample size to be at least 8,000. Sample sizes varied between 8,158 for the Netherlands and 12,828 for Bulgaria. All countries followed the centrally developed questionnaire, with some national specific adjustments. Face-to-face interviews were conducted; in Russia, Bulgaria, and Georgia interviewers recorded the interviews on paper, and in France, Germany, and the Netherlands by using computer-assisted methods (CAPI). All data sets were cleaned according to specific guidelines and this was followed by a centralized harmonizing phase. Country data sets, however, varied due to- among other things-local differences in the fieldwork design and performance and in data gathering methods,

Measuring instruments

The De Jong Gierveld Loneliness Scale (De Jong Gierveld and Van Tilburg 2006), as used in the GGS, encompassed three negatively formulated items ("I experience a general sense of emptiness", "I miss having people around" and "Often, I feel rejected") and three positively formulated items ("There are plenty of people that I can lean on in case of trouble", "There are many people that I can count on completely" and "There are enough people that I feel close to"). None of the items referred directly to loneliness and the word loneliness has not been used in the set of items. The items had three response categories: "no," "more or less" and "yes." Around 1985, in developing the scale, the item response model of Rasch was applied: scale scores were based on dichotomous item scores with the answer "more or less" always indicating loneliness (De Jong Gierveld and Kamphuis 1985). Processing the scale data entailed counting neutral and positive answers ("more or less", "yes") on negatively formulated items. This resulted in the emotional loneliness score, ranging from 0 (not emotionally lonely) to 3 (intensely emotionally lonely). Counting neutral and negative answers ("no" and "more or less") on the positively formulated items resulted in the social loneliness score, ranging from 0 to 3 (intensely socially lonely). Loneliness scale scores were not computed when item scores were missing.

To identify partner status, the following question was formulated: "I would like to ask you about all persons who live in this household. Who are they? To help me keep track of your answers, please tell me how they are related to you." A show card categorized 18 types of relationships to the respondent, with "partner or spouse" included as the first one. Children living in the household were identified using the "child" type of relationship. In addition, the following question was asked: "Have you given birth to/fathered any other children, or have you ever adopted any other children who do not currently live in your household?" The question measuring health was: "How is your health in general?" with five answer categories ranging from "very poor" to "very good." The evaluation of the financial situation of a respondent's household was elicited by asking: "Thinking of your household's total monthly income is your household able to make ends meet?" with six answer categories ranging from "with great difficulty" to "very easily".

\section{Procedure}

The reliability and validity of the emotional and social loneliness scales have been tested and presented separately 
for older adults and younger adults. We arbitrarily made a choice for the age of 60 as the cut off point, distinguishing between older people aged 60 to 79 years and younger adults aged 18 to 59 years.

The postulated existence of two dimensions-emotional and social loneliness - has been examined by means of confirmatory factor analysis incorporated in the LISREL 8 program (Jöreskog and Sörbom 1993). Since item scores were dichotomous tetrachoric correlations were computed and Weighted Least Squares (WLS) estimation was applied. Country specific estimates were computed, followed by testing whether factor loadings were invariant over the seven countries. Multigroup confirmatory factor analyses tested whether the factor structure underpinning the six items was similar for all countries under investigation by holding the factor loadings in the model invariant across countries. We adopted the procedure and evaluation criteria for model fit recommended by $\mathrm{Hu}$ and Bentler (1998, 1999). More specifically, we applied the combinational rules of Standardized Root Mean Square Residual $(\mathrm{SRMR}) \leq 0.08$ (an absolute index of the fit between obtained and implied covariance matrices) and the Comparative Fit Index (CFI) $\geq 0.95$ (a noncentrality-based index computed as a function of chi-square, degrees of freedom, and sample size). The LISREL program was also applied to compute the reliability of the emotional and social loneliness scales.

The invariance of regression coefficients of age, gender, partner status, number of children, subjective health, and the evaluation of the household financial situation-computed by means of LISREL, based on Pearson correlations and adopting maximum likelihood estimation, has been investigated across the seven countries; there was no constraint on the nonrecursive effect between emotional and social loneliness. The evaluation of the household financial situations of respondents in the Netherlands and in Japan was unknown and, therefore, the analyses were conducted for the other predictors within seven countries and for all predictors within five countries.

\section{Results}

Descriptive analysis of responses

Table 2 provides information about the scores on the loneliness scales: scores in any one country differed from the scores in other countries, for the older as well as for the younger adults. Among older adults, both Georgia and Bulgaria were characterized by high loneliness scores on the emotional as well as the social scale, with Russia following in third place. Scores were much lower in the three Western European countries, with Japanese respondents in a middle position between Western and Eastern European respondents. Scores for respondents aged 18-59 years were considerably lower than among older adults. At the level of the items, interesting variations have been shown. The percentages of (both younger and older) respondents agreeing with the item "Often, I feel rejected" were among the lowest in six of the seven countries. The item "There are many people that I can count on completely" was not endorsed by a high percentage of respondents in any of the seven countries (both for older and younger adults), indicating that the social network of most respondents was restricted to a small number of confidants. Furthermore, most respondents agreed with the item "There are enough people I feel close to"; especially among adults aged 18-59 years. It seems that in all countries, respondents state that they are surrounded by "not many", but "enough" people that made them feel embedded. However, country differences in loneliness are prevalent.

The mean age of respondents in the older adults group was around 68 years, with the exception of Japan, i.e., the country where respondents were selected only up to 69 years of age. Subjective health of older adults proved to be better in Japan and the Western European countries than in Eastern European countries. For the adults aged 18 to 59 years, subjective health proved to be better in Japan, followed by the Western European countries and Bulgaria, then Russia and Georgia. Table 2 also shows sharp differences between Eastern and Western European countries in the financial situation of households, both for the older adults and for the adults aged 18 to 59 years.

Testing the reliability of the two scales

Reliability coefficients support the good psychometric characteristics of the two loneliness scales. For the measurement of emotional loneliness reliability for older adults was 0.81 (in France) or higher, while for social loneliness for older adults the coefficients were 0.85 (in France) or higher (see Table 3). For younger adults, the outcomes of the reliability tests were similar.

Tests on the two-factor structure

Information about the division of the items according to the two postulated underlying factors of the scale in each of the countries under investigation is shown in Table 4. Within the confirmatory factor analysis, the six items were categorized into the subscales as postulated. The correlation between the two latent factors proved to be moderate in most countries and high in France and Germany as far as the outcomes for the older adults group are concerned. For the younger group, the correlations between the two latent 
Table 2 Descriptives of sample (Means and proportions)

\begin{tabular}{|c|c|c|c|c|c|c|c|c|}
\hline & France & Germany & Netherlands & Russia & Bulgaria & Georgia & Japan & \\
\hline \multicolumn{9}{|l|}{$N:$} \\
\hline Age $60-79$ (age $60-69$ for Japan) & 2,541 & 2,560 & 1,565 & 2,804 & 2,470 & 2,266 & 1,891 & \\
\hline Sex (Female) & .55 & .50 & .56 & .69 & .50 & .60 & .50 & $\mathrm{Chi}_{(6)}^{2}=325$ \\
\hline Age (60-79 years) & 68.72 & 68.23 & 68.05 & 68.91 & 68.13 & 69.20 & 64.31 & $F_{(6,16090)}=200$ \\
\hline Partner in household & .58 & .62 & .58 & .43 & .67 & .58 & .85 & $\mathrm{Chi}_{(6)}^{2}=922$ \\
\hline Number of children $(0-12)$ & 2.23 & 1.72 & 2.46 & 1.80 & 1.80 & 2.36 & 1.98 & $F_{(6,16037)}=117$ \\
\hline Subjective health (1-5, very good) & 3.52 & 3.45 & 3.78 & 2.49 & 2.98 & 2.43 & 4.22 & $F_{(6,16045)}=1446$ \\
\hline Financial situation (1-6, very easily) & 3.80 & 4.17 & & 2.12 & 1.85 & 1.89 & & $F_{(4,12567)}=3077$ \\
\hline Emotional loneliness (0-3) & .75 & .63 & .65 & .98 & 1.14 & 1.48 & .63 & $F_{(6,16090)}=804$ \\
\hline Experience emptiness & .33 & .19 & .25 & .44 & .39 & .56 & .23 & $\mathrm{Chi}_{(6)}^{2}=1065$ \\
\hline Miss people around & .29 & .29 & .25 & .32 & .40 & .54 & .17 & $\mathrm{Chi}_{(6)}^{2}=790$ \\
\hline Feel rejected & .13 & .16 & .15 & .21 & .35 & .38 & .22 & $\mathrm{Chi}_{(6)}^{2}=781$ \\
\hline Social loneliness $(0-3)$ & .95 & 1.07 & 1.24 & 1.73 & 2.08 & 2.27 & 1.33 & $F_{(6,16090)}=469$ \\
\hline People in case of trouble ${ }^{a}$ & .31 & .40 & .35 & .60 & .70 & .81 & .48 & $\mathrm{Chi}_{(6)}^{2}=1940$ \\
\hline Can count on many people ${ }^{\mathrm{a}}$ & .41 & .37 & .52 & .65 & .76 & .84 & .58 & $\mathrm{Chi}_{(6)}^{2}=1808$ \\
\hline Enough close people ${ }^{\mathrm{a}}$ & .23 & .31 & .38 & .49 & .62 & .62 & .27 & $\mathrm{Chi}_{(6)}^{2}=1551$ \\
\hline \multicolumn{9}{|l|}{$N:$} \\
\hline Age $18-59$ & 7,514 & 6,970 & 5,754 & 8,398 & 10,273 & 7,734 & 7,009 & \\
\hline Sex (Female) & .57 & .56 & .59 & .60 & .56 & .55 & .54 & $\mathrm{Chi}_{(6)}^{2}=95$ \\
\hline Age (18-59 years) & 39.58 & 39.23 & 40.45 & 38.75 & 36.52 & 38.12 & 40.63 & $F_{(6,53651)}=130$ \\
\hline Partner in household & .61 & .63 & .67 & .64 & .67 & .66 & .69 & $\mathrm{Chi}_{(6)}^{2}=139$ \\
\hline Number of children $(0-12)$ & 1.45 & 1.24 & 1.38 & 1.37 & 1.26 & 1.52 & 1.93 & $F_{(6,51900)}=247$ \\
\hline Subjective health (1-5, very good) & 4.06 & 4.10 & 4.09 & 3.31 & 4.06 & 3.51 & 4.38 & $F_{(6,53567)}=1855$ \\
\hline Financial situation (1-6, very easily) & 3.39 & 3.82 & & 2.43 & 2.23 & 2.39 & & $F_{(4,40661)}=2928$ \\
\hline Emotional loneliness $(0-3)$ & .60 & .57 & .48 & .71 & .72 & .88 & .67 & $F_{(6,53652)}=120$ \\
\hline Experience emptiness & .25 & .17 & .18 & .33 & .26 & .36 & .24 & $\mathrm{Chi}_{(6)}^{2}=1045$ \\
\hline Miss people around & .24 & .23 & .17 & .22 & .25 & .31 & .18 & $\mathrm{Chi}_{(6)}^{2}=548$ \\
\hline Feel rejected & .11 & .17 & .13 & .16 & .21 & .21 & .25 & $\mathrm{Chi}_{(6)}^{2}=758$ \\
\hline Social loneliness $(0-3)$ & .93 & .95 & 1.04 & 1.45 & 1.76 & 1.87 & 1.28 & $F_{(6,53652)}=846$ \\
\hline People in case of trouble ${ }^{a}$ & .28 & .32 & .25 & .49 & .58 & .67 & .50 & $\mathrm{Chi}_{(6)}^{2}=4464$ \\
\hline Can count on many people ${ }^{a}$ & .45 & .34 & .47 & .56 & .66 & .75 & .56 & $\mathrm{Chi}_{(6)}^{2}=3513$ \\
\hline Enough close people ${ }^{a}$ & .20 & .29 & .32 & .41 & .53 & .45 & .22 & $\mathrm{Chi}_{(6)}^{2}=3140$ \\
\hline
\end{tabular}

${ }^{a}$ Proportion of respondents not agreeing with the item

Note: For all comparisons country differences are significant at $p<.001$

Table 3 Reliability of the 3-item loneliness scales

\begin{tabular}{llllllll}
\hline & France & Germany & Netherlands & Russia & Bulgaria & Georgia & Japan \\
\hline$N$ : & & & & & & & \\
Age 60-79 & 2,541 & 2,560 & 1,565 & 2,804 & 2,470 & 2,266 & 1,891 \\
$\quad$ Emotional loneliness & 0.81 & 0.85 & 0.88 & 0.86 & 0.91 & 0.87 & 0.86 \\
$\quad$ Social loneliness & 0.85 & 0.91 & 0.88 & 0.88 & 0.95 & 0.90 & 0.90 \\
$N$ : & & & & & & & \\
Age 18-59 & 7,514 & 6,970 & 5,754 & 8,398 & 10,273 & 7,734 & 7,009 \\
$\quad$ Emotional loneliness & 0.82 & 0.83 & 0.90 & 0.86 & 0.89 & 0.87 & 0.87 \\
Social loneliness & 0.85 & 0.92 & 0.88 & 0.88 & 0.94 & 0.87 & 0.90 \\
\hline
\end{tabular}

factors proved to be moderate for the Eastern European countries and Japan, and higher for the three Western European countries.
The results of the analyses showed model fit for each of the countries under investigation, both for the older adults and for the adults aged 18 to 59 years, indicating that the 
Table 4 Confirmative factor analysis of emotional and social loneliness items

\begin{tabular}{|c|c|c|c|c|c|c|c|}
\hline & France & Germany & Netherlands & Russia & Bulgaria & Georgia & Japan \\
\hline \multicolumn{8}{|l|}{$N:$} \\
\hline Age $60-79$ & 2,541 & 2,560 & 1,565 & 2,804 & 2,470 & 2,266 & 1,891 \\
\hline \multicolumn{8}{|c|}{ Factor loadings for emotional loneliness } \\
\hline Experience emptiness & 0.73 & 0.86 & 0.84 & 0.87 & 0.93 & 0.84 & 0.89 \\
\hline Miss people around & 0.86 & 0.71 & 0.91 & 0.80 & 0.78 & 0.82 & 0.88 \\
\hline Feel rejected & 0.70 & 0.85 & 0.77 & 0.80 & 0.93 & 0.85 & 0.69 \\
\hline \multicolumn{8}{|c|}{ Factor loadings for social loneliness } \\
\hline People in case of trouble & 0.79 & 0.84 & 0.78 & 0.84 & 0.91 & 0.87 & 0.86 \\
\hline Can count on many people & 0.85 & 0.93 & 0.86 & 0.90 & 0.98 & 0.88 & 0.94 \\
\hline Enough close people & 0.80 & 0.88 & 0.89 & 0.80 & 0.88 & 0.84 & 0.78 \\
\hline \multicolumn{8}{|l|}{ Model fit } \\
\hline $\mathrm{CFI}$ & 0.99 & 1.00 & 1.00 & 1.00 & 1.00 & 1.00 & 1.00 \\
\hline SRMR & 0.058 & 0.042 & 0.040 & 0.057 & 0.029 & 0.043 & 0.045 \\
\hline Correlation between factors & 0.64 & 0.68 & 0.47 & 0.43 & 0.36 & 0.48 & 0.50 \\
\hline \multicolumn{8}{|l|}{$N:$} \\
\hline Age $18-59$ & 7,514 & 6,970 & 5,754 & 8,398 & 10,273 & 7,734 & 7,009 \\
\hline \multicolumn{8}{|c|}{ Factor loadings for emotional loneliness } \\
\hline Experience emptiness & 0.76 & 0.81 & 0.90 & 0.86 & 0.90 & 0.85 & 0.89 \\
\hline Miss people around & 0.82 & 0.69 & 0.86 & 0.75 & 0.74 & 0.79 & 0.85 \\
\hline Feel rejected & 0.75 & 0.86 & 0.85 & 0.85 & 0.92 & 0.87 & 0.76 \\
\hline \multicolumn{8}{|c|}{ Factor loadings for social loneliness } \\
\hline People in case of trouble & 0.81 & 0.87 & 0.81 & 0.84 & 0.91 & 0.82 & 0.87 \\
\hline Can count on many people & 0.82 & 0.92 & 0.85 & 0.89 & 0.95 & 0.87 & 0.97 \\
\hline Enough close people & 0.79 & 0.87 & 0.87 & 0.80 & 0.89 & 0.82 & 0.76 \\
\hline \multicolumn{8}{|l|}{ Model fit } \\
\hline CFI & 0.99 & 1.00 & 1.00 & 1.00 & 1.00 & 1.00 & 1.00 \\
\hline SRMR & 0.051 & 0.032 & 0.037 & 0.050 & 0.008 & 0.051 & 0.041 \\
\hline Correlation between factors & 0.70 & 0.64 & 0.66 & 0.38 & 0.32 & 0.45 & 0.51 \\
\hline
\end{tabular}

emotional and the social scales have proven to be two solid dimensions of the overarching loneliness concept. For both the older adults and respondents aged 18-59 years, the test of the invariance of factor loadings failed ( $\mathrm{df}=80$; $\mathrm{SRMR}=0.36 ; \quad \mathrm{CFI}=0.93 ; \quad \mathrm{df}=80 ; \quad \mathrm{SRMR}=0.37$; $\mathrm{CFI}=0.93$; respectively), indicating that correlations between items differ across the countries. For example, among older adults factor loadings in France are relatively low and the correlation between the factors is high, indicating that emotional and social loneliness items share meaning. In contrast, factor loadings in Bulgaria are high and the correlation between the factors is relatively low, indicating that emotional and social loneliness items are distinguished more sharply. However, the results of all country specific analyses supported the two-dimensional measurement of loneliness.

\section{Prediction of loneliness}

In order to investigate the congruent validity of the two loneliness scales, several variables that have been shown in previous studies to be significantly associated with emotional and/or social loneliness were tested here for invariance over the seven countries, using LISREL multiple group model testing. The regression coefficients for the predictors of emotional and of social loneliness are estimated as equal over the countries. In other words, despite the country differences in these variables as shown in Table 2 the outcomes in terms of emotional and social loneliness are similar for individuals with similar characteristics living in different countries. The results of this procedure are shown in Table 5; for older adults and younger adults, respectively. Since regression effects are estimated as similar across the seven counties $t$-tests for significance are based on the pooled data set with the combined sample size. Significant regression coefficients were shown for partner status in association with emotional loneliness: for the older adults -0.24 and for adults aged 18 to 59 years -0.19 (both in Model 2). These coefficients indicate the importance of having a spouse or partner for the alleviation of emotional loneliness in adults of all ages, but especially so among those aged 60 and over. Partner 
Table 5 Regression of loneliness (standardized coefficients)

\begin{tabular}{|c|c|c|c|c|c|c|}
\hline & \multicolumn{3}{|c|}{ Age $60-79$} & \multicolumn{3}{|c|}{ Age $18-59$} \\
\hline & Model 1 & Model 2 & Model 3 & Model 1 & Model 2 & Model 3 \\
\hline$N$ & 16,097 & 16,097 & 12,641 & 53,652 & 53,652 & 40,889 \\
\hline \multicolumn{7}{|l|}{ Emotional loneliness } \\
\hline Sex (Female) & $0.09 *$ & 0.00 & 0.01 & $0.06^{*}$ & $0.04 *$ & $0.05 *$ \\
\hline Age & $0.10^{*}$ & 0.02 & $0.03 *$ & $0.08 *$ & $0.04 *$ & $0.06 *$ \\
\hline Partner in household & & $-0.24 *$ & $-0.23 *$ & & $-0.19 *$ & $-0.17 *$ \\
\hline Number of children & & $-0.04 *$ & $-0.04^{*}$ & & $0.02 *$ & -0.01 \\
\hline Subjective health & & $-0.21^{*}$ & $-0.18^{*}$ & & $-0.22 *$ & $-0.19 *$ \\
\hline Financial situation & & & $-0.14^{*}$ & & & $-0.16^{*}$ \\
\hline \multicolumn{7}{|l|}{ Social loneliness } \\
\hline Sex (Female) & $-0.05^{*}$ & $-0.08^{*}$ & $-0.06^{*}$ & $-0.03^{*}$ & $-0.05^{*}$ & $-0.03 *$ \\
\hline Age & $0.05^{*}$ & 0.02 & 0.02 & $0.12 *$ & $0.08 *$ & $0.09 *$ \\
\hline Partner in household & & $-0.07^{*}$ & $-0.08^{*}$ & & $-0.06^{*}$ & $-0.04 *$ \\
\hline Number of children & & $-0.12 *$ & $-0.14^{*}$ & & 0.01 & -0.02 \\
\hline Subjective health & & $-0.13^{*}$ & $-0.11^{*}$ & & $-0.14 *$ & $-0.11 *$ \\
\hline Financial situation & & & $-0.13 *$ & & & $-0.15^{*}$ \\
\hline \multicolumn{7}{|l|}{ Model fit } \\
\hline df & 24 & 60 & 48 & 24 & 60 & 48 \\
\hline SRMR & 0.066 & 0.043 & 0.020 & 0.057 & 0.025 & 0.011 \\
\hline CFI & 0.95 & 0.97 & 0.98 & 0.95 & 0.99 & 0.99 \\
\hline
\end{tabular}

$* P<0.001$

Note: Estimates and statistics for the multiple group model with pooled data; Model 1 and 2 for seven countries; Model 3 for five countries

status proved to be significant also for social loneliness, but the coefficients are lower. Additionally, the regression coefficients showed that social loneliness of older adults is significantly correlated with the number of children (beta $=-0.12$ in Model 2). Older adults with no children, or with a small number of children, have a higher risk of social loneliness than older adults with more children in such a situation. The number of children of adults aged 18-59 years of age is not significantly associated with social loneliness.

The results presented in Table 5 also support congruent validity in that older and younger adults in less than optimal health are significantly more at risk of emotional and of social loneliness in the countries under investigation (regression coefficients are -0.18 and -0.11 for emotional loneliness and social loneliness, respectively, in Model 2). The associations between emotional and social loneliness and difficulties in making ends meet in the household, also proved to be significant for the five countries for which we have data. Furthermore, there is unequivocal support for the expected association that there are gender and age differences in loneliness. Among older people women are more intensely emotionally lonely than males (Model 1), which can be understood by the fact that women less often have a partner, are more often in poor health and more often have difficulties in making ends meet (Model 2). However, males have more intense feelings of social loneliness, which holds when we control for other individual characteristics in Model 2. Women aged between 18 and 59 are more intensely emotionally lonely than men but again the reverse is observed for social loneliness. This gender difference holds when other individual characteristics are controlled (Model 2). Among the older adults, the oldest are more intensely emotionally and socially lonely. To a large extent, however, age differences are to be understood through differences in partner and parental status, health, and financial position. Among younger respondents, the oldest are more intensely lonely than younger respondents. Across the various regression models the effects of partner and (for social loneliness) parental status, health, and financial poison are relatively strong and for gender and age relatively small, indicating that there is a large variability in loneliness within the categories of women and men, and within age categories.

\section{Discussion}

This article aimed at testing the psychometric characteristics of the 3-item emotional and the 3-item social loneliness 
scales included in the Generations and Gender Surveys as used in six European countries and Japan, both for adults aged 60 and over, and for adults aged 18-59 years. The findings showed that the emotional and social loneliness can be measured well using the 3-item emotional and the 3-item social loneliness scale. Both the scales displayed good reliability coefficients. Using confirmatory factor analyses, the existence of the emotional and social loneliness scales has been confirmed for each of the seven countries under investigation. The results of the strong test on invariance of factor loadings across the countries showed that the contrast between emotional and social loneliness differed. Within the regression analysis predicting differences in loneliness we, therefore, accepted that the correlation of emotional and social loneliness varied across the countries.

As part of the validity testing, the scales were investigated by multivariate LISREL regression analyses for the associations with six well-known loneliness-related risk variables. Older and younger respondents without a partner scored significantly higher on the emotional loneliness scale than other respondents. Missing the partner as attachment person, missing the daily structuring element of a partner in the household and missing the partner as mediator of the size and functioning of the social network, was shown to be a risk factor for emotional loneliness (conforming with Leung et al. 2008; Pinquart and Sörensen 2001). It was shown that children are important in the context of social loneliness: contact with them is seen as a major pathway toward social integration of older adults (Buber and Engelhardt 2008). This role of facilitating integration into the broader community is connected to the specific functioning of grown-up children: awareness of the risk of social isolation of their parents may lead adult children to support them by being good listeners. It is supposed that this role is in general beyond the capacities of younger children. We did not observe a relationship between number of children and social loneliness among younger respondents (who often have dependent children). Additionally, the risks of emotional and social loneliness proved to be closely related to a shortage of resources in both the younger and older adults, for example as far as their health and financial condition is concerned: these restrictions hamper the giving of support and care, intensify the feelings of stress and deprivation and consequently affect both aspects of loneliness, be it emotional or social. Moreover, after controlling for the effects of age, partner status, health, and financial situation, women proved to be significantly less socially lonely than their male peers; this was shown both for older as well as for younger respondents. In midlife an optimal social functioning-be it in intimate relationships or in the broader social context-is fostered by women's abilities to take the initiative in organizing contacts with kin and non-kin. In this investigation, as an outcome of the LISREL multivariate regression analyses, it was found that emotional and social loneliness were significantly, but modestly associated with age of respondents. Based on previous research (De Jong Gierveld et al. 2009) we expected that effects of age areto a certain extent-mediated via network characteristics, poor health conditions and a shortage of financial means. The outcomes of the tests on congruent validity did not invalidate these considerations. In general, our observations did support congruent validity of both the emotional and the social loneliness scale for adults aged 18-79 years.

The population-based samples-representing the population of men and women of each of the birth cohorts between 18 and 79 years of age-as well as the large sample sizes of the GGS surveys in all countries under investigation are important in guaranteeing the usefulness of the scales for the general population of each of the countries, including the older segment of the population. In conclusion, these test outcomes indicated that the 3-item De Jong Gierveld emotional and social loneliness scales are reliable and valid scales for use in differing types of countries. The short versions of these scales are shown to possess satisfactory psychometric properties, and are attractive for reasons of cost effectiveness and in terms of validity and reliability for researchers who wish to adopt a loneliness measuring instrument in large-scale surveys.

Nevertheless the data have weaknesses. Data per country were collected via different data collection modalities, i.e. paper-and-pencil and CAPI-procedures. Future research has to investigate the effects of these differences in fieldwork situations. Additionally, it should be noted that for the investigation of the validity of the two scales only a limited number of loneliness-related variables were considered in the analyses. The size, composition, and functioning of the social network, religious bonds, participation in volunteer activities, other bonds within the local community, norms and values regarding solidarity, and support exchanges within families, as well as societal characteristics and interaction effects of country and individual characteristics (De Jong Gierveld et al. 2006), are among the many indicators known to protect against loneliness but were not incorporated in this analysis. The GGS is characterized by a panel design. However, at the time of this study only the first wave of data was available for analysis and therefore we did not test the long-term stability of emotional and social loneliness scores as measured in these countries. However, the qualities of the 3-item emotional and the 3-item social loneliness scales as investigated and confirmed in this study broaden the possibilities for research into loneliness in the older, and also in the younger population, be it in small-scale or large-scale surveys, using data from countries that differ significantly in their country-level and individual characteristics. 
Acknowledgments We thank the Japanese GGP Committee for their permission to include the Japanese loneliness items in this article. The data for the Netherlands were drawn from the Netherlands Kinship Panel Study, which is funded by grant 480-10-009 from the Major Investments Fund of the Netherlands Organization for Scientific Research (NWO), and by the Netherlands Interdisciplinary Demographic Institute (NIDI), Utrecht University, University of Amsterdam and Tilburg University.

Open Access This article is distributed under the terms of the Creative Commons Attribution Noncommercial License which permits any noncommercial use, distribution, and reproduction in any medium, provided the original author(s) and source are credited.

\section{References}

Buber I, Engelhardt H (2008) Children's impact on the mental health of their older mothers and fathers: findings from the Survey of Health, Ageing and Retirement in Europe. Eur J Ageing 5:31-45

Carstensen LL (1992) Social and emotional patterns in adulthood: support for Socioemotional Selectivity Theory. Psychol Aging 7:331-338

Cornwell EY, Waite LJ (2009) Measuring social isolation among older adults using multiple indicators from the NSHAP Study. J Gerontol Soc Sci 64B(suppl 1):i38-i46

De Jong Gierveld J, Kamphuis F (1985) The development of a Raschtype loneliness scale. Appl Psychol Meas 9:289-299

De Jong Gierveld J, Van Tilburg TG (1999) Manual of the loneliness scale. VU University, Amsterdam

De Jong Gierveld J, Van Tilburg TG (2006) A six-item scale for overall, emotional and social loneliness: confirmative tests on new survey data. Res Aging 28:582-598

De Jong Gierveld J, Van Tilburg TG, Dykstra PA (2006) Loneliness and social isolation. In: Vangelisti A, Perlman D (eds) Cambridge handbook of personal relationships. Cambridge University Press, Cambridge, pp 485-500

De Jong Gierveld J, Broese van Groenou M, Hoogendoorn AW, Smit $\mathrm{JH}$ (2009) Quality of marriages in later life and emotional and social loneliness. J Gerontol Soc Sci 64B:497-506

Dykstra PA (2009) Older adult loneliness: myths and realities. Eur J Ageing 6:91-100

Dykstra PA, De Jong Gierveld J (1994) The theory of mental incongruity, with a specific application to loneliness among widowed men and women. In: Erber R, Gilmour R (eds) Theoretical frameworks for personal relationships. Erlbaum, Hillsdale N.J., pp 235-259

Dykstra PA, Fokkema T (2007) Social and emotional loneliness among divorced and married men and women: comparing the deficit and cognitive perspectives. Basic Appl Soc Psychol 29: $1-12$

Hawkley LC, Hughes ME, Waite LJ, Masi CM, Thisted RA, Cacioppo JT (2008) From social structural factors to perceptions of relationship quality and loneliness: the Chicago health, aging, and social relations study. J Gerontol Soc Sci 63B:S375-S384

$\mathrm{Hu}$ LT, Bentler PM (1998) Fit indices in covariance structure modeling: sensitivity to underparameterized model misspecification. Psychol Methods 3:424-453

$\mathrm{Hu}$ LT, Bentler PM (1999) Cutoff criterion for fit indices in covariance structure analysis: conventional criteria versus new alternatives. Struct Equ Model 6:1-55

Jöreskog KG, Sörbom D (1993) LISREL 8 user's reference guide. SSI, Chicago, IL

Korporaal M, Broese van Groenou M, Van Tilburg TG (2008) Effects of own and spousal disability on loneliness among older adults. J Aging Health 20:306-325

Leung GTY, De Jong Gierveld J, Lam LCW (2008) Validation of the Chinese translation of the 6-item De Jong Gierveld loneliness scale in elderly Chinese. Int Psychogeriatr 20:1262-1272

Penninx BWJH, Van Tilburg TG, Kriegsman DMW, Boeke AJP, Deeg DJH, Van Eijk JTM (1999) Social network, social support, and loneliness in older persons with different chronic diseases. $\mathbf{J}$ Aging Health 11:151-168

Perlman D, Peplau LA (1981) Toward a social psychology of loneliness. In: Gilmour R, Duck S (eds) Personal relationships 3: personal relationships in disorder. Academic, London, pp 31-43

Pinquart M (2003) Loneliness in married, widowed, divorced, and never-married older adults. J Soc Pers Rel 20:31-53

Pinquart M, Sörensen S (2001) Influences on loneliness in older adults: a meta-analysis. Basic Appl Soc Psychol 23:245-266

Savikko N, Routasalo PE, Tilvis RS, Strandberg TE, Pitkala KH (2005) Predictors and subjective causes of loneliness in an aged population. Arch Gerontol Geriatr 41:223-233

Stevens N, Westerhof GJ (2006) Marriage, social integration and loneliness in the second half of life: a comparison of Dutch and German men and women. Res Aging 28:713-729

Van Tilburg TG (1998) Losing and gaining in old age: changes in personal network size and social support in a four-year longitudinal study. J Gerontol Soc Sci 53B:S313-S323

Vikat A, Spéder Z, Beets G, Billari FC, Bühler C, Désesquelles A et al (2007) Generations and gender programme: concepts and guidelines. United Nations, New York and Geneva

Waite LJ, Gallagher M (2000) The case for marriage: why married people are happier, healthier and better off financially. Doubleday, New York

Weiss RS (1973) Loneliness: the experience of emotional and social isolation. The MIT Press, Cambridge, MA 